\title{
JAHN-TELLER AND HERZBERG-TELLER COUPLINGS IN ABSORPTION AND MAGNETIC CIRCULAR DICHROISM SPECTRA OF $(E+E) * e$ SYSTEMS
}

\author{
M. PILCh aNd M. PaWlikowski \\ Department of Theoretical Chemistry, Jagiellonian University \\ Karasia 3, 30-060 Kraków, Poland
}

(Received May 13, 1992)

\begin{abstract}
Model calculations are reported for an $(E+E) * e$ system including linear and quadratic Jahn-Teller and Herzberg-Teller vibronic interactions. The effects of these interactions on magnetic circular dichroism and absorption spectra are compared with experimental observations on $\mathrm{CoF}_{6}^{3-}$ salts.
\end{abstract}

PACS numbers: 33.10.Lb, 33.55.Be

\section{Introduction}

The problem of magnetic circular dichroism (MCD) for degenerate and non-degenerate electronic transitions has been extensively studied during the past three decades [1-4]. Most of the theoretical papers concerning MCD for degenerate transitions dealt with the electronic origin of the excited state magnetic moments and their calculation by quantum-chemical methods. The vibronic structure of MCD spectra was often ignored or solved within the Franck-Condon (FC) and Jahn-Teller (JT) approximations. However, recently Zgierski has shown that a proper understanding of MCD structures for non-degenerate transitions requires a theory that goes beyond the FC approximation [4].

While the JT and FC approximations offer good starting points to study vibronic details of MCD and absorption spectra, they may be insufficient for highly symmetrical molecules such as transition metal complexes. The electronic states of these molecules, e.g., those resulting from $d-d$ and $d-p$ electron promotions, are energetically close to one another and vibronic couplings can arise not only within degenerate states, but also between electronic states of different parentage. In molecules belonging to $D_{3}\left(D_{3 h}\right)$ and $D_{3 d}$ point groups a double-degenerate dipole-allowed $E\left(E^{\prime}\right)$ state can be vibronically coupled to $E\left(E^{\prime}\right)$ and $A_{2}\left(A_{2}^{\prime}\right)$ states via vibrational modes of $e\left(e^{\prime}\right)$-symmetry. Thus, we can have a situation where 
$(E+E) * e$ vibronic coupling is present, e.g., two $E\left(E^{\prime}\right)$ states subject to the JT effect can be involved in Herzberg-Teller (HT) coupling via a JT-active vibrational mode. The vibronic theory for the $(E+A) * e$ coupling scheme has been reported and discussed in great detail for molecules of trigonal, pentagonal, and hexagonal symmetry [5]. This paper addresses certain theoretical aspects of MCD and absorption in a situation where the $(E+E) * e$ coupling scheme applies.

Specifically, we discuss MCD and absorption spectra corresponding to $A \rightarrow E$ electronic excitation in molecules of $D_{3}\left(D_{3 h}\right)$ symmetry. In particular, we wish to show that the Jahn-Teller effect considered exclusively within the $E * e$ coupling scheme [6] may be insufficient to account correctly for absorption and MCD spectra in the region corresponding to $A \rightarrow E$ electronic excitation. Thus, the intensity distribution observed in the absorption spectra of certain $\mathrm{CoF}_{6}^{3-}$ salts, interpreted as due to a dynamic JT effect [7], is at variance with the simple coupling scheme. To show this while rendering the presentation as simple as possible, we consider a single $e\left(e^{\prime}\right)$ vibrational mode and two electronic states involved in Jahn-Teller coupling. These two states together with their vibronic manifolds are allowed to interact by Herzberg-Teller couplings. Vibronic couplings are assumed to be linear and quadratic in the coordinates of the double-degenerate mode. Therefore, the theory formulated here and applied to molecules of $D_{3}\left(D_{3 h}\right)$ symmetry can be immediately adapted to study MCD for molecules of $D_{3}\left(D_{3 h}, D_{3 d}\right)$, $D_{5}\left(D_{5 h}, D_{5 d}\right)$, and $D_{6}\left(D_{6 h}, D_{6 d}\right)$ symmetry.

\section{Model formulation}

We consider a molecule of $D_{3}\left(D_{3 h}\right)$ symmetry with a single double-degenerate harmonic vibration represented by complex coordinates $Q_{ \pm}$and momenta $\widehat{P}_{ \pm}$. In the harmonic approximation, the degenerate vibrational mode can be fully characterized by the algebraic properties of two commuting operators

$$
\begin{aligned}
& \widehat{h}_{0}=\widehat{P}_{+} \widehat{P}_{-}+\omega^{2} Q_{+} Q_{-}, \\
& \hat{L}_{z}=\mathrm{i}\left(Q_{+} \widehat{P}_{+}-Q_{-} \widehat{P}_{-}\right) .
\end{aligned}
$$

Here $\widehat{h}_{0}$ and $\widehat{L}_{z}$ represent the vibrational hamiltonian and angular momentum operator, respectively; both are given in units $\hbar=1$. With the help of the well-known commutator relations $\left[Q_{ \pm}, \widehat{P}_{ \pm}\right]=\mathrm{i}$ and $\left[Q_{\mp}, \widehat{P}_{ \pm}\right]=0[8]$, we can easily find the algebraic relationships

$$
\left[\widehat{L}_{z}, Q_{ \pm}^{p}\right]= \pm 2^{p-1} Q_{ \pm}^{p},\left[\widehat{L}_{z}, \widehat{P}_{ \pm}^{p}\right]=\mp 2^{p-1} \widehat{P}_{ \pm}^{p}
$$

which hold for any integer $p$.

Now let two excited singlet electronic states be represented by complex electronic functions $\Phi_{m \pm}^{0}$ and $\Phi_{n \pm}^{0}$ given in the ground state nuclear equilibrium configuration $Q_{ \pm}=0$. The diabatic functions $\Phi_{m \pm}^{0}$ and $\Phi_{n \pm}^{0}$ belong to the $E\left(E^{\prime}\right)$ representation, i.e., the corresponding states are paramagnetic and dipole-allowed from the ground electronic state $\Phi_{0}^{0}$. The electronic electric $\widehat{D}$ and magnetic $\widehat{M}$ dipole operators thus obey selection rules

$$
\left\langle\Phi_{0}^{0}|\hat{D}| \Phi_{k \pm}^{0}\right\rangle=2^{-1 / 2} D_{k}\left(e_{x} \pm \mathrm{i} e_{y}\right),
$$




$$
\left\langle\Phi_{k \pm}^{0}|\widehat{M}| \Phi_{k \pm}^{0}\right\rangle= \pm M_{k k} \boldsymbol{e}_{z},
$$

as follows immediately from symmetry arguments applied to molecules of $D_{3}\left(D_{3 h}\right)$ symmetry. The matrix elements $D_{k}$ and $M_{k, k}$ for $k=m$ and $n$ represent, respectively, the transition dipole moment and intrinsic magnetic moment of the $k$-th state.

The use of the diabatic representation implies that the excited electronic states are vibronically coupled via $Q$-dependent terms in the electronic hamiltonian $\widehat{H}_{\mathrm{e}}(q, Q)$ of the molecule [9]. To take this coupling into account we express the total (vibronic) wave functions, in agreement with the variational principle, as

$$
\Psi_{v, \lambda}=\Phi_{m-}^{0} \Lambda_{v, \lambda}^{m-}+\Phi_{m+}^{0} \Lambda_{v, \lambda}^{m+}+\Phi_{n-}^{0} \Lambda_{v, \lambda}^{n-}+\Phi_{n+}^{0} \Lambda_{v, \lambda}^{n+} .
$$

The index $v$ numbers the vibronic levels and $\lambda$ refers to their symmetry. For $\lambda=0$ the vibronic functions (4) are of $A_{1}\left(A_{1}^{\prime}\right)$ or $A_{2}\left(A_{2}^{\prime}\right)$ symmetry and those for $\lambda=+$ and $\lambda=-$ belong to the $E\left(E^{\prime}\right)$ representation of the $D_{3}\left(D_{3 h}\right)$ point group. The vibrational components $\Lambda_{v, \lambda}^{\alpha}$ satisfy the vibronic equation

$$
\left(\widehat{\mathcal{H}}-E_{v, \lambda}\right)\left\{\Lambda_{v, \lambda}^{m-}, \Lambda_{v, \lambda}^{m+}, \Lambda_{v, \lambda}^{n-}, \Lambda_{v, \lambda}^{n+}\right\}=0,
$$

where $\{\ldots\}$ denotes a column vector and $E_{v, \lambda}$ is the energy corresponding to (4). The hamiltonian $\widehat{\mathcal{H}}$ is represented by a $4 \times 4$ matrix and consists of elements defined as

$$
\begin{aligned}
& \widehat{\mathcal{H}}_{k \pm k^{\prime} \pm}=\left\langle\Phi_{k \pm}^{0}\left|\widehat{H}_{\mathrm{e}}(q, Q)\right| \Phi_{k^{\prime} \pm}^{0}\right\rangle+\delta_{k k^{\prime}} \widehat{P}_{+} \widehat{P}_{-}, \\
& \widehat{\mathcal{H}}_{k \pm k^{\prime} \mp}=\left\langle\Phi_{k \pm}^{0}\left|\widehat{H}_{\mathrm{e}}(q, Q)\right| \Phi_{k^{\prime} \mp}^{0}\right\rangle .
\end{aligned}
$$

The leading terms in these expressions depend parametrically on the vibrational coordinates; their functional forms can be derived from symmetry as Longuet-Higgins showed [10]. Therefore, expanding $\widehat{H}_{\mathrm{e}}(q, Q)$ in a Taylor's series of $Q_{ \pm}$and retaining only quadratic and lower terms, we can express $\widehat{\mathcal{H}}$ in terms of Dirac's matrices $\sigma_{i}$ and $\rho_{i}[11]$ as

$$
\begin{aligned}
\widehat{\mathcal{H}}= & {\left[\widehat{P}_{+} \widehat{P}_{-}+\omega^{2} Q_{+} Q_{-}+\frac{1}{2}\left(E_{m}+E_{n}\right)\right] 1+\frac{1}{2}\left(E_{m}-E_{n}\right) \rho_{3} } \\
& +\left(\sigma_{1}+\mathrm{i} \sigma_{2}\right)\left(\Gamma_{1} Q_{-}+\Gamma_{2} Q_{+}^{2}\right)+\left(\sigma_{1}-\mathrm{i} \sigma_{2}\right)\left(\Gamma_{1} Q_{+}+\Gamma_{2} Q_{-}^{2}\right),
\end{aligned}
$$

where $E_{m}$ and $E_{n}$ are the diabatic potential energies at $Q_{ \pm}=0$. The matrices

$$
\begin{aligned}
& \Gamma_{1}=\frac{1}{2}\left[\left(k_{m}+k_{n}\right) 1+\left(k_{m}-k_{n}\right) \rho_{3}+k \rho_{1}\right], \\
& \Gamma_{2}=\frac{1}{2}\left[\left(g_{m}+g_{n}\right) 1+\left(g_{m}-g_{n}\right) \rho_{3}+g \rho_{1}\right],
\end{aligned}
$$

are due to linear $\left(\Gamma_{1}\right)$ and quadratic $\left(\Gamma_{2}\right)$ couplings. Applying the explicit forms of Dirac's matrices [11], one can easily see that the first two terms in $\Gamma_{1}$ and $\Gamma_{2}$ introduce vibronic coupling in the $m$-th and $n$-th electronic states. The constants $k_{p}$ and $g_{p}$ for $p=m$ and $n$ are thus referred to as linear and quadratic JT coupling parameters, respectively. The third terms in (8) are responsible for the vibronic mixing of different electronic states. Thus, $k$ and $g$ are called linear and quadratic HT coupling constants, respectively. 
In the next section the theory formulated above is applied to MCD and absorption spectra within the Buckingham and Stephens theory of the MCD phenomenon [1]. Thus, the MCD signal and absorption intensity for a vibronic transition from totally symmetric state $\Psi_{00}\left(=\Phi_{0}^{0} \eta_{00}\left(Q_{+}, Q_{-}\right)\right)$are characterized by two Faraday parameters $A_{1}$ and $B_{0}$ and an absorption coefficient $D_{0} . A_{1}$ and $D_{0}$ are defined as $[3,9]$ :

$$
\begin{aligned}
& A_{1}(0 \rightarrow v)=\operatorname{Im} \sum_{\lambda} \sum_{\lambda^{\prime}}\left\langle\Psi_{v \lambda^{\prime}}|\widehat{\boldsymbol{M}}| \Psi_{v \lambda}\right\rangle \cdot\left\langle\Psi_{00}|\widehat{D}| \Psi_{v \lambda^{\prime}}\right\rangle \times\left\langle\Psi_{v \lambda}|\widehat{D}| \Psi_{00}\right\rangle, \\
& D_{0}(0 \rightarrow v)=\sum_{\lambda}\left\langle\Psi_{00}|\widehat{D}| \Psi_{v \lambda}\right\rangle \times\left\langle\Psi_{v \lambda}|\widehat{D}| \Psi_{00}\right\rangle
\end{aligned}
$$

The parameter $B_{0}$ implied herein is given in Ref. [8]. The MCD and absorption spectra are calculated from expressions

$$
\begin{aligned}
& \frac{\Delta A}{\nu}=\eta\left[A_{1}\left(-\frac{\partial f}{\partial \nu}\right)+\left(B_{0}+\frac{C_{0}}{k T}\right) f\right] \beta H, \\
& \frac{A}{\nu}=\eta D_{0} f
\end{aligned}
$$

The symbols in Eqs. (10) have their usual meaning [3] and $f(\nu)$ is normalized to unity intensity distribution of the individual absorption line assumed to be Gaussian.

\section{Results and discussion}

Before presenting exact results based on numerical calculations, we define an operator

$$
\widehat{\mathcal{L}}=\widehat{L}_{z} 1+\Xi \sigma_{3}
$$

which represents the total (vibronic) angular momentum. Now let us consider the commutator

$$
\begin{aligned}
& {[\widehat{\mathcal{H}}, \widehat{\mathcal{L}}]=(2 \Xi-1) \Gamma_{1}\left[\left(\sigma_{1}-\mathrm{i} \sigma_{2}\right) Q_{+}-\left(\sigma_{1}+\mathrm{i} \sigma_{2}\right) Q_{-}\right]} \\
& +2(\Xi-1) \Gamma_{2}\left[\left(\sigma_{1}-\mathrm{i} \sigma_{2}\right) Q_{-}^{2}-\left(\sigma_{1}+\mathrm{i} \sigma_{2}\right) Q_{+}^{2}\right]
\end{aligned}
$$

which can be readily obtained from (2) when the familiar commutator-anticommutator relations [11] for $\sigma_{i}$ and $\rho_{i}$ are applied. Since for $E\left(E^{\prime}\right)$ states of molecules of $D_{3}\left(D_{3 h}\right)$ symmetry $\Xi$ equals $1 / 2[6,10]$, the commutator $(12)$ vanishes in the absence of quadratic coupling, i.e., when $\Gamma_{2}=0$. In that case $\widehat{\mathcal{H}}$ and $\widehat{\mathcal{L}}$ have the same sets of wave functions characterized by angular momentum quantum numbers: $j= \pm 1 / 2, \pm 3 / 2, \pm 5 / 2, \ldots$ etc. Except for the levels with $j= \pm 3 / 2, \pm 9 / 2, \pm 15 / 2$, ... etc., which are accidentally degenerate, although belong to non-degenerate representations, all levels are double-degenerate and can support the magnetic moments measured in MCD experiments. However, the strongly restrictive selection rule permits only transitions to vibronic levels with $j= \pm 1 / 2$ from the totally symmetric ground state [10], so that only those states contribute to MCD and absorption. The same restriction applies when three and more excited states involved in linear JT and HT coupling give rise to MCD and absorption spectra. 


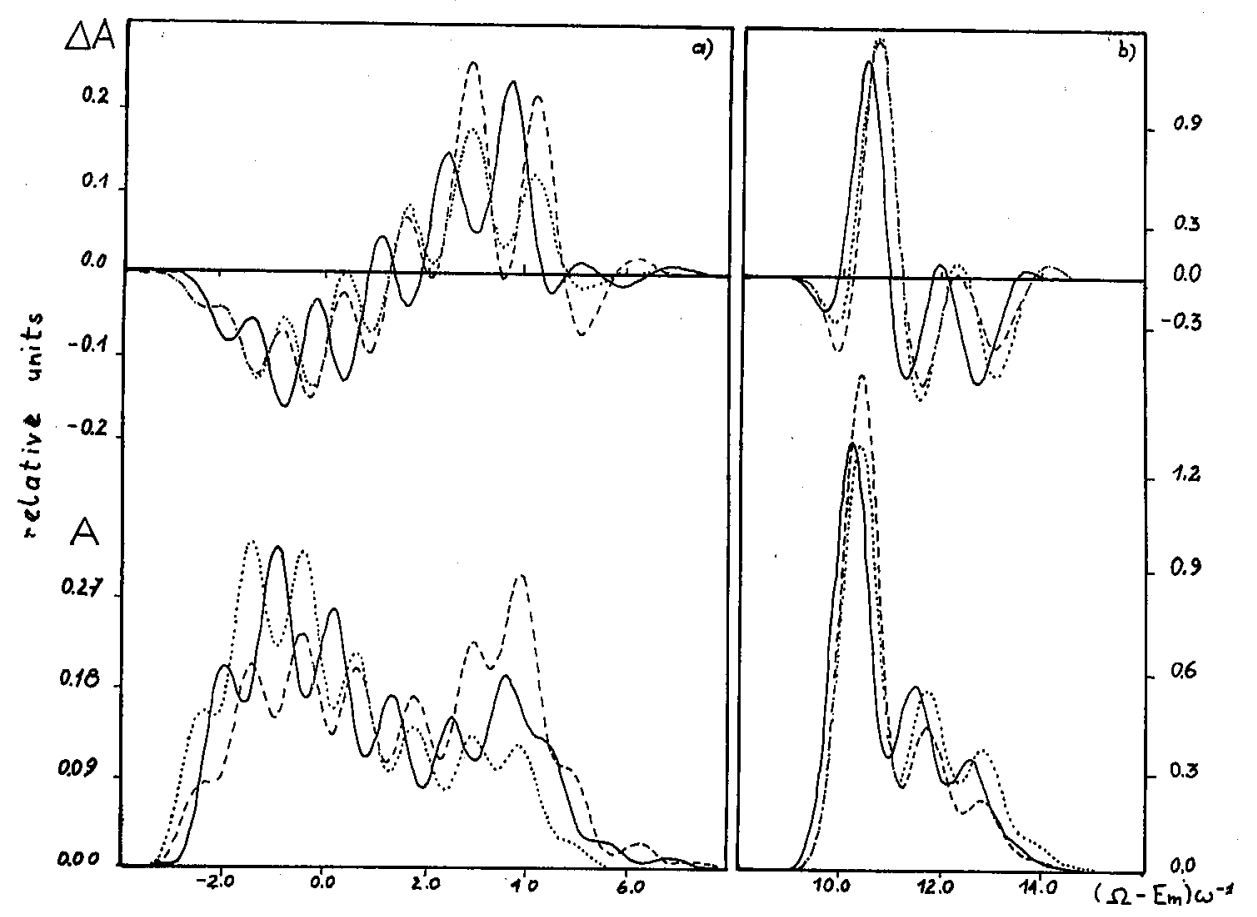

Fig. 1. MCD and absorption spectra calculated for (a) $\Phi_{0}^{0} \rightarrow \Phi_{m \pm}^{0}$ and (b) $\Phi_{0}^{0} \rightarrow \Phi_{n \pm}^{0}$ electronic transitions. The parameters are $E_{n}-E_{m}=10 \omega, M_{m}=-M_{n}=\beta, D_{m} / D_{n}=$ $0.15, k_{m}=\sqrt{5} \omega^{3 / 2}, k_{n}=\omega^{3 / 2}, k=0(-), k=\omega^{3 / 2}(\cdots \cdots), k=-\omega^{3 / 2}(----)$; the line width $\Gamma=0.5 \omega$.

Then the proposed theory amounts to a logical extension of that formulated by Longuet-Higgins [10].

Figure 1 presents MCD and absorption spectra calculated for $\Phi_{0}^{0} \rightarrow \Phi_{m \pm}$ (Fig. 1a) and $\Phi_{0}^{0} \rightarrow \Phi_{n \pm}^{0}$ (Fig. 1b) electronic excitations and for different values of the linear HT coupling constant. The parameters $g_{m}, g_{n}$ and $g$ are set equal to zero. The solid line spectra correspond to the situation where states $\Phi_{m \pm}^{0}$ and $\Phi_{n \pm}^{0}$ are not vibronically coupled, i.e., $k=0$. Then the absorption spectrum of $\Phi_{0}^{0} \rightarrow \Phi_{m \pm}^{0}$ electronic transition shows the distinctive two-band structure expected when an $E$ state suffers a large JT distortion [6]. Figure 1a shows that linear HT coupling tends to suppress the two-band character of absorption such that one of two bands can be almost eliminated for certain values of the HT parameter. At the same time the intensity distribution of MCD and absorption spectra of $\Phi_{0}^{0} \rightarrow \Phi_{n \pm}^{0}$ remains practically intact as shown in Fig. 1b.

The remarkable behavior of the absorption spectrum illustrated in Fig. 1a can be qualitatively understood in terms of an adiabatic ("static") approximation to the exact solutions of Eq. (5). The adiabatic functions needed to evaluate the transition moments have the form (4) but the vibrational coefficients satisfy Eq. (5) 
with the kinetic energy operator $\widehat{P}_{+} \widehat{P}_{-}$omitted [12]. In this approximation, which is justified if $k_{m}$ and $k_{n}$ are not very small, Eq. (5) is exactly solvable in the absence of quadratic coupling and yields

$$
\begin{aligned}
& \Phi_{m \pm}=2^{-1 / 2}\left[\left(\Phi_{m-}^{0} \cos \theta_{ \pm}+\Phi_{n-}^{0} \sin \theta_{ \pm}\right) \mathrm{e}^{-\mathrm{i} \phi / 2}\right. \\
& \left.\mp\left(\Phi_{m+}^{0} \cos \theta_{ \pm}+\Phi_{n+}^{0} \sin \theta_{ \pm}\right) \mathrm{e}^{\mathrm{i} \phi / 2}\right] \\
& E_{m \pm}(Q)=\frac{1}{2}\left\{\omega^{2} \rho^{2} \mp\left[\left(k_{m}+k_{n}\right)+\left(k_{m}-k_{n}\right) \cos \theta_{ \pm}+k \sin \theta_{ \pm}\right] \rho\right\}
\end{aligned}
$$

where

$$
\tan 2 \theta_{ \pm}= \pm 2 k \rho /\left[E_{n}-E_{m} \mp\left(k_{n}-k_{m}\right) \rho\right],
$$

$\rho$ and $\phi$ are polar coordinates of the double-degenerate vibration. The adiabatic functions $\Phi_{m+}$ and $\Phi_{m-}$ correspond, respectively, to the low- and high-energy bands as can be deduced from the adiabatic potentials $E_{m \pm}(Q)$. Using (13) to calculate the transition dipole moments, we get for the $\Phi_{0} \rightarrow \Phi_{m-}$ transition

$$
\left\langle\Phi_{0}|D| \Phi_{m-}\right\rangle=2^{1 / 2}\left(D_{m} \cos \theta_{-}+D_{n} \sin \theta_{-}\right)\left(e_{x} \sin \phi / 2+e_{y} \cos \phi / 2\right)
$$

and for the $\Phi_{0} \rightarrow \Phi_{m+}$ transition

$$
\left\langle\Phi_{0}|\boldsymbol{D}| \Phi_{m+}\right\rangle=2^{1 / 2}\left(D_{m} \cos \theta_{+}+D_{n} \sin \theta_{+}\right)\left(e_{x} \cos \phi / 2-e_{y} \sin \phi / 2\right) .
$$

Inspection of Eqs. (14) and (15) reveals that HT coupling will always upset the intensity of the low- or high-energy band in the absorption associated with a strongly JT-distorted state. The effect will be strong when the $\Phi_{m \pm}^{0}$ state is coupled to an electronic state with a substantially larger transition dipole n.oment, i.e., when a strong inequality $D_{m} \ll D_{n}$ holds. In such a case, the low-energy band is enhanced whereas the high-energy band is reduced for positive values of the linear HT coupling parameter $k$. The opposite holds for negative values of $k$ as shown in Fig. 1a. It should be noted that expressions (14) and (15) are incomplete and have to be vibrationally averaged with a weighting factor given by the product of the initial and final state vibrational wave functions. The resulting intensity of individual (vibronic) transitions is then also strongly dependent on the HT coupling. Such a dependence is apparent for small energy separations, $E_{n}-E_{m}$, of the coupled states and for large differences, $k_{n}-k_{m}$, between the JT parameters. These effects are included in our exact numerical treatment, but an explicit discussion is omitted in the interest of brevity.

The changes in absorption depicted in Fig. 1a are accompanied by substantial changes in the MCD signal, especially in the high-energy tails of MCD spectra. To get a better insight into these spectra, the magnetic moments of individual $\Psi_{v}(j=1 / 2)$ states were calculated as functions of the HT coupling parameter. The results given in terms of $A_{1} / D_{0}$ ratios are presented in Fig. 2. Since $A_{1} / D_{0}$ ratios are even functions of $k$, only curves for positive $k$ are given and numbered in order of increasing energy $v=1,2,3 \ldots$

Figure 2 shows that drastic changes of the magnetic moments are expected in the high-energy part of the MCD spectra which are due to a substantial alteration of the magnetic moments for the $\Psi_{6}(j=1 / 2)$ and $\Psi_{7}(j=1 / 2)$ levels. The energies of these states are close to the conical intersection point of the two adiabatic 


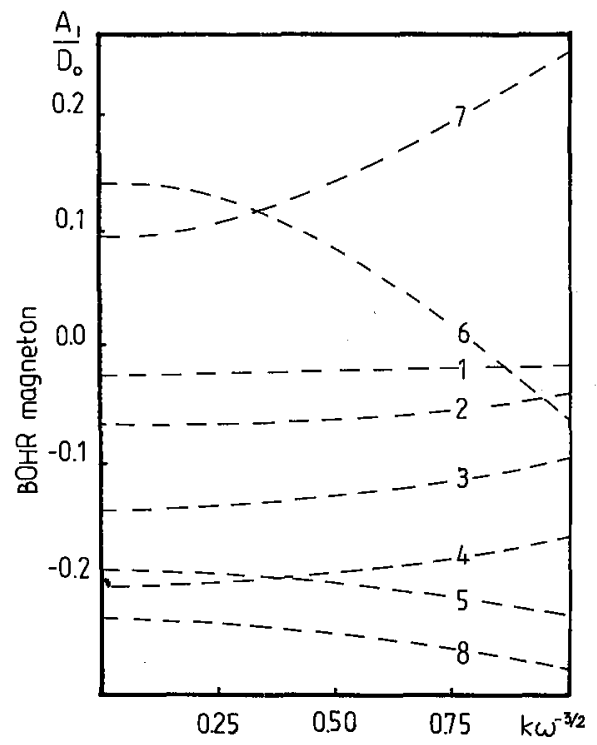

Fig. 2. MCD $A_{1} / D_{0}$ ratios as functions of $k$. The other parameters as in Fig. 1.

potential surfaces at $Q_{ \pm}=0$. The magnetic moment changes are thus due to non-adiabatic effects in the vicinity of the intersection point.

Thus far, we have assumed that quadratic terms are absent from Eq. (5), so that the commutator (12) vanishes and $j$ is a good quantum number. When both linear and quadratic couplings are present, the commutator does not vanish and the levels with different $j$ are mixed via the quadratic term in the Hamiltonian (7). This mixing involves all levels, but those with $j= \pm 1 / 2, \pm 5 / 2, \pm 7 / 2, \pm 11 / 2$, ...etc. give rise to dipole-allowed states of $E\left(E^{\prime}\right)$ symmetry. Since the mixed levels carry also magnetic moments, the increase in the density of states due to quadratic coupling can affect not only absorption, but also MCD spectra. That increase in state density will tend to smooth out the MCD and absorption spectra as shown in Fig. 3 for not very large value of RT coupling constant in the $\Phi_{m \pm}^{0}$ state. For large values of HT and RT coupling constant the situation may be much more complicated since not only intensity distributions but also energy vibronic levels are expected to be strongly modified. We hope to return to study these problems in one of the future papers.

The examples of calculations presented in this paper allow us to conclude that MCD and absorption spectra corresponding to $A \rightarrow E$ electronic excitations can differ sometimes substantially from those calculated in the framework of $E * e$ JT coupling theory [6], namely when the excited $E\left(E^{\prime}\right)$ state interacts vibronically with other electronic $E\left(E^{\prime}\right)$ states via a JT active vibration. We have shown that the absorption spectrum corresponding to a strongly JT distorted $E$ state may loose its distinctive two-band profile. In particular, the high-energy band in absorption may be much more intense than the low-energy band. Such a reversed 


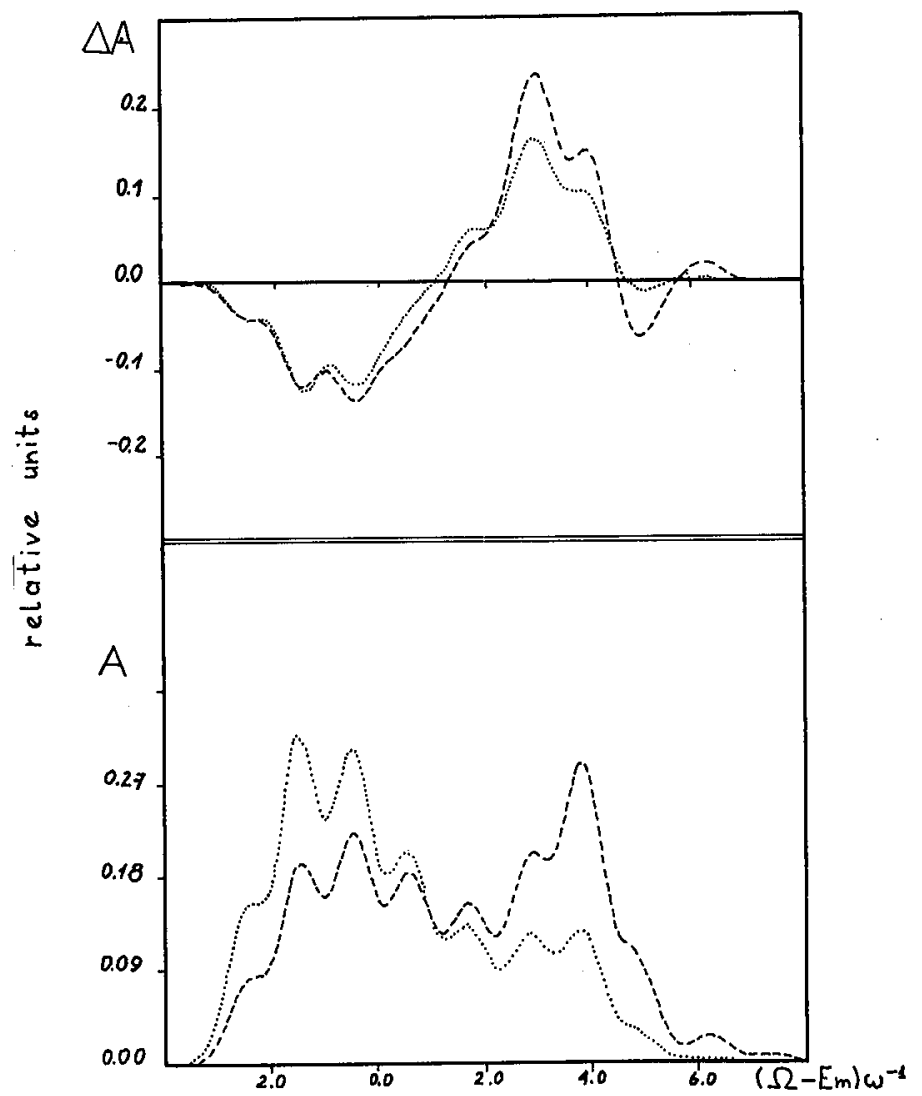

Fig. 3. MCD and absorption spectra calculated for (a) $\Phi_{0}^{0} \rightarrow \Phi_{m}^{0}$ transition. The parameters are the same as in Fig. 1 except for $g_{m}=0.75 \omega, k=-\omega^{-3 / 2}(---)$ and $g_{m}=0.75 \omega, k=\omega^{-3 / 2}(\cdots \cdots)$.

intensity pattern has been observed in the electronic reflection spectra of several $\mathrm{CoF}_{6}^{3-}$ salts [12]. According to Cotton and Meyers [7], the two components observed for these (near-) cubic systems are due to a dynamic JT effect. The relative intensities of these two components are at variance with a simple $E * e$ coupling scheme, but can be explained if HT coupling between different $E$ states is included. Since the spectra show no resolved vibrational structure and since no MCD spectra are available, a more debated analysis is not possible at this time.

The present theory is also relevant to the MCD and absorption spectra of sym-trichlorobenzene, $\mathrm{C}_{6} \mathrm{H}_{3} \mathrm{Cl}_{3}$ [13]. For that molecule the prominent MCD band at $c a .49000 \mathrm{~cm}^{-1}$ probably consists of two transitions corresponding to closely spaced $E_{1 \mathrm{u}}$ and $E_{2 \mathrm{~g}}$ states of benzene [14]. However, in order to describe the MCD spectra for $\mathrm{C}_{6} \mathrm{H}_{3} \mathrm{Cl}_{3}, B_{1 \mathrm{u}}$ and $B_{2 \mathrm{u}}$ benzene-like states must be included. This application will be presented elsewhere. 


\section{Acknowledgement}

We thank for a grant from Jagiellonian University for support of this work. The special thanks are to Dr. W. Siebrand for reading the manuscript and for his critical remarks.

\section{References}

[1] A.D. Buckingham, P.J. Stephens, Ann. Rev. Phys. Chem. 17, 399 (1966).

[2] P.J. Stephens, Ann. Rev. Phys. Chem. 25, 201 (1974).

[3] P.J. Stephens, Adv. Chem. Phys. 35, 197 (1976).

[4] M.Z. Zgierski, J. Chem. Phys. 85, 109 (1986) and references therein.

[5] M.Z. Zgierski, M. Pawlikowski, J. Chem. Phys. 70, 3444 (1979).

[6] H.C. Longuet-Higgins, V. Öpik, M.H.L. Price, R.A. Sack, Proc. R. Soc. (London) A 244, 1 (1958).

[7] F.A. Cotton, M.D. Meyers, J. Am. Chem. Soc. 82, 5023 (1960).

[8] M. Pawlikowski, T.A. Keiderling, J. Chem. Phys. 81, 4765 (1984).

[9] W. Siebrand, M.Z. Zgierski, in: Excited States, Vol. 4, Ed. E.C. Lim, Academic, New York 1979, p. 1.

[10] H.C. Longuet-Higgins, Adv. Spectrosc. 2, 429 (1961).

[11] P.A.M. Dirac, The Principles of Quantum Mechanics, Oxford University Press, London 1958, p. 257.

[12] R. Englman, The Jahn-Teller Effect in Molecules and Crystals, Wiley Intersc., London 1972.

[13] A. Kaito, A. Tajiri, M. Hatano, Chem. Phys. Lett. 25, 548 (1974).

[14] A.M. Taleb, I.H. Munro, J.B. Birks, Chem. Phys. Lett. 21, 454 (1973). 